\title{
A Single Institutional Experience with Panitumumab in Metastatic Colorectal Cancer
}

\author{
Sigurdis Haraldsdottir*, Jeffrey S. Rose, Christina Wu, Lai Wei, Richard M. Goldberg, \\ Tanios Bekaii-Saab
}

The Ohio State University Comprehensive Cancer Center, Columbus, USA.

Email: " sigurdis.haraldsdottir@osumc.edu

Received October $1^{\text {st }}, 2012$; revised October $31^{\text {st }}, 2012$; accepted November $10^{\text {th }}, 2012$

\begin{abstract}
Introduction: Panitumumab is an EGFR inhibitor approved for use in metastatic refractory colorectal cancer. It is unclear whether patients who have progressed on cetuximab may benefit from subsequent panitumumab therapy. This retrospective analysis was conducted to describe the experience at The Ohio State University with panitumumab including in patients who have progressed on cetuximab. Methods: Patients who received at least 1 dose of panitumumab between September 2006 and December 2011 were identified using the hospital's pharmacy database. Response Evaluation Criteria in Solid Tumors (RECIST) 1.1 was used to assess responses and Kaplan-Meier curves were used to estimate progression-free survival (PFS) and overall survival (OS). Results: Eighty-seven patients (median age 61 years) were identified. Sixty-seven percent of patients had tumors with wild-type KRAS, 3.4\% had tumors with mutated KRAS and the KRAS status was unknown in $29.9 \%$. Twenty-four percent of the patients had an ECOG performance status of 2 or above and $59.8 \%$ of patients had received $\geq 2$ prior lines of chemotherapy. Thirty-two percent of patients received single-agent panitumumab while $68 \%$ received it in combination with chemotherapy. Of the patients with KRAS wild-type tumors, $10(17.2 \%)$ had objective responses (3 complete, 7 partial) and 26 (44.8\%) had stable disease. Median PFS and OS were 5.0 and 9.0 months. The presence of a rash, improved ECOG performance status and coadministration with either irinotecan or FOLFIRI, led to a significantly better OS in univariate analysis. Among patients who had clinical benefit with cetuximab, $71 \%$ had subsequent clinical benefit with panitumumab therapy. Conclusions: In our single institution analysis of patients who received panitumumab, the number of prior lines of therapy did not significantly affect OS, suggesting that panitumumab retains its efficacy in the 2nd and 3rd line setting. Additionally, panitumumab can benefit patients who previously had clinical benefit with cetuximab.
\end{abstract}

Keywords: Panitumumab; Cetuximab; EGFR Inhibitor; Metastatic Colorectal Cancer

\section{Introduction}

Colorectal cancer is the 2nd leading cause of cancerrelated death in the United States with approximately 50,000 annual deaths [1]. About $20 \%$ of the patients present with metastatic disease and the 5-year overall survival with treatment is $\sim 10 \%$ [2]. Cytotoxic agents such as 5-Fluorouracil (5-FU), oxaliplatin and irinotecan are well established in the treatment of metastatic colorectal cancer (mCRC) and have in recent years been combined with targeted therapies, which have become an important component of systemic therapy.

Panitumumab is a fully humanized monoclonal $\mathrm{IgG}-2$ antibody targeting epidermal growth factor receptor (EGFR) and was approved by the Food and Drug Administration (FDA) in September 2006 as therapy in mCRC. Cetuximab, a chimeric monoclonal IgG-1 antibody, was FDA-

"Corresponding author. approved in 2004. EGFR is a $170 \mathrm{kDa}$ receptor tyrosine kinase, and a member of the human epidermal family. Its overexpression or constitutive action has been shown to affect signaling cascades in carcinogenesis, most importantly the RAS/RAF/MAPK pathway. The RAS proteins are serine-threonine kinases that are activated downstream of EGFR. The mutational status of KRAS was shown to predict responses to EGFR-targeted therapy in a study published in 2006 [3], and thus KRAS mutation testing became a NCCN recommendation in November 2008.

Single agent panitumumab in patients with KRAS wild-type tumors has been shown to have an objective response rate (ORR) of $17 \%$ and to improve progression-free survival (PFS) and overall survival (OS) compared to best supportive care [4]. When given with 5-FU and irinotecan (FOLFIRI) in the second-line setting, panitumumab improves ORR by $\sim 20 \%$, and PFS and OS 
by about 2 months when compared to FOLFIRI alone [5]. When panitumumab is combined with 5-FU and oxaliplatin (FOLFOX) as first-line therapy, it has been shown to improve PFS by 1.6 months and OS by $\sim 4$ months [6] which differs from cetuximab which has not been shown to improve efficacy consistently (and has never been shown to improve overall survival) when given with FOLFOX [7-9]. This combination has been removed from NCCN guidelines.

It is unclear whether panitumumab has activity in patients who have previously progressed on cetuximab. Two prospective studies have had discrepant results. Metges et al. reported responses in $22 \%$ of patients on single-agent panitumumab where $34 \%$ of patients had previously had responses on cetuximab. [10]. Wadlow et al. published a phase II trial of 20 patients in which no responses were seen but $45 \%$ patients had stable disease with a median PFS of 1.7 months and median OS of 5.2 months [11]. A retrospective review of 15 patients with progression on cetuximab revealed a minor radiographic response in 3 patients, and stable disease in 3 patients after 8 weeks of single-agent panitumumab [12]. In another retrospective study among 22 patients who had been on cetuximab (the majority did not have progression on therapy), 9 patients had a minor or partial response [13].

The goals of this retrospective study were to: 1) Assess the ORR, PFS and OS in all patients treated with panitumumab at a tertiary medical center; 2) To assess responses to panitumumab in patients previously treated with cetuximab.

\section{Methods and Materials}

\subsection{Design}

This study was a single-institution retrospective analysis of patients diagnosed with mCRC who were treated with panitumumab. The study was approved by The Ohio State University Internal Review Board. The study included all patients with histologically proven mCRC treated with panitumumab from June 2006 to December 2011. Patient data was collected using the information warehouse and pharmacy database of the James Cancer Hospital. Patients on the study had to have received at least 1 dose of panitumumab and have measurable disease. One cycle was considered 1 month of therapy (therapy given every 2 weeks).

\subsection{Evaluation of Response}

Response Evaluation Criteria in Solid Tumors (RECIST) 1.1 was used to evaluate treatment response as assessed by computed tomography [14]. Radiographic reports were accessed and tumor measurements documented. Biochemi- cal response was assessed using tumor marker carcinoembryonic antigen (CEA) measurements when available, before and after treatment. Adverse events, where available, were recorded based on definitions from the National Cancer Institute Common Toxicity Criteria, version 4.0.

PFS was calculated from the start of panitumumab therapy to clinical or radiological progression or death from any cause, whichever occurred first. Patients who did not meet these criteria were censored at the last date panitumumab was given. OS was calculated from the start of panitumumab until the date of death from any cause. Patients still alive were censored on the date of their last follow-up.

\subsection{Statistical Analysis}

Descriptive statistics (mean, standard deviation, range for continuous variables and frequency for categorical variables) were provided to describe the patient population. The SAS for Windows ${ }^{\circledR}$ Version 9.2 software was used. Survival curves were estimated using the method of Kaplan-Meier and compared using the log-rank test. Estimated median survival with $95 \%$ confidence intervals $(95 \% \mathrm{CI})$ was provided. Covariates identified as having an influence on survival by univariate analysis (p-value $<$ 0.1 ) were analyzed using the Cox proportional hazards model. The step-down regression method was used to build the final statistical models. Statistical significance was determined at $\mathrm{p}<0.05$.

\section{Results}

\subsection{Demographics and Tumor Characteristics}

A total of 87 patients were identified to have received panitumumab from June 2006 until December 2011. Patient and tumor characteristics are shown in Table 1. Sixty-three patients $(72.4 \%)$ had a colon primary, 23 patients $(26.4 \%)$ had a rectal primary and in one case $(1.2 \%)$ the location of the primary site was unclear. Tumor grade was well-differentiated in 5 tumors $(7.2 \%)$, moderately differentiated in 43 tumors (61.4\%) and poorly differentiated in 22 tumors $(31.4 \%)$. All of the tumors were adenocarcinomas; 8 tumors had mucinous features, 3 tumors had signet ring cell features and 2 tumors had neuroendocrine features with $\mathrm{Ki}-67$ of $>90 \%$ and $80 \%$. BRAF mutation status was assessed in $6(6.9 \%)$ tumors: 3 tumors had the V600E mutation and 3 tumors were BRAF wild-type. Microsatellite instability was assessed in $17(19.5 \%)$ tumors by immunohistochemistry, 15 tumors had intact mismatch repair protein staining while 2 tumors were found to lack mismatch repair protein expression. KRAS mutation status was not assessed in any patients starting panitumumab prior to June 2008 and 
Table 1. Patient demographics and tumor characteristics.

\begin{tabular}{lc}
\hline Median age, years (range) & $61(26-84)$ \\
\hline Sex & Number $(\%)$ \\
Males & $46(52.9 \%)$ \\
Females & $41(47.1 \%)$ \\
\hline Race & \\
Caucasian & $76(87.4 \%)$ \\
African American & $9(10.4 \%)$ \\
Hispanic & $1(1.1 \%)$ \\
Asian & $1(1.1 \%)$ \\
\hline
\end{tabular}

\begin{tabular}{lc}
\hline Site of disease & \\
Liver limited & $18(20.7 \%)$ \\
1 site (other than liver) & $13(15.0 \%)$ \\
Peritoneal spread & $15(17.2 \%)$ \\
$>1$ site & $41(47.1 \%)$ \\
\hline ECOG Performance status & \\
0 & $16(18.4 \%)$ \\
1 & $50(57.5 \%)$ \\
2 & $18(20.7 \%)$ \\
3 & $3(3.4 \%)$ \\
\hline KRAS & \\
Wild-type & $58(66.7 \%)$ \\
Mutated & $3(3.4 \%)$ \\
Unknown & $26(29.9 \%)$
\end{tabular}

Number of previous chemotherapy regimens

$\begin{array}{lc}\text { None } & 5(5.7 \%) \\ 1 & 30(34.5 \%) \\ 2 & 36(41.4 \%) \\ 3 \text { or more } & 16(18.4 \%)\end{array}$

Panitumumab administration

\begin{tabular}{lc} 
Single agent & $28(32.2 \%)$ \\
With FOLFIRI & $40(46.0 \%)$ \\
With irinotecan & $13(14.9 \%)$ \\
With FOLFOX & $3(3.4 \%)$ \\
With capecitabine & $2(2.3 \%)$ \\
With CAPIRI & $1(1.2 \%)$ \\
\hline
\end{tabular}

$\mathrm{N}=87$.

was therefore unknown in $26(29.9 \%)$ patients. Three patients were classified as having tumors with KRAS mutations as follows: 1 patient was found to have discrepant results, with KRAS G12/13 mutation found in the primary rectal tumor but not on the bone metastasis; 1 patient had a tumor with a G12S KRAS mutation; and the third patient had a tumor with an atypical KRAS mu- tation in codon 8 (V8I) within exon 2 which is of unknown significance.

Forty-three (49.4\%) patients had previously been exposed to oxaliplatin- and irinotecan-based chemotherapy, $28(32.2 \%)$ patients to irinotecan-based chemotherapy and $11(12.6 \%)$ patients to oxaliplatin-based chemotherapy. Seventy-four $(85.1 \%)$ patients had received bevacizumab previously and 17 (19.5\%) cetuximab previously.

\subsection{Efficacy}

Of the 58 patients who were known to have KRAS wild-type tumors, 10 (17.3\%) had an ORR (see Table 2) with 3 complete responses and 7 partial responses. A total of $36(62.1 \%)$ patients with KRAS wild-type tumors had a clinical benefit while on panitumumab. All of the complete responses and 4 of the partial responses were seen in patients on FOLFIRI/panitumumab, 2 partial responses were in patients on single-agent panitumumab and 1 partial response in a patient on irinotecan/panitumumab therapy. No responses were seen in patients with tumors with unknown KRAS mutational status.

In the KRAS wild-type patients, 54 patients had available data on CEA measurements and 11 (20.4\%) patients did not have pretreatment CEA elevations. Of those who did, $29(67.4 \%)$ patients had a decrease in CEA after panitumumab initiation (mean decrease $62 \%$ $+/-29)$ while $14(32.6 \%)$ patients did not (mean increase $155 \%+/-208)$.

The median PFS was 3.0 months (95\% CI 3.0 - 5.0) and the median OS was 8.0 months (95\% CI 5.0 - 11.0) in all patients. PFS was significantly higher in patients with KRAS wild-type tumors versus tumors with unknown KRAS status (5.0 months (95\% CI 3.0 - 6.0) versus 2.5 months $(95 \%$ CI $2.0-4.0) ; \mathrm{p}=0.008)$, respectively. Similarly, OS was significantly better in patients with KRAS wild-type tumors versus tumors with unknown KRAS status (9.0 months (95\% CI 5.0 - 14.0) versus 6.5 months (95\% CI $3.0-9.0)$; $\mathrm{p}=0.026)$, respectively (see Figure 1).

Table 2. Efficacy of panitumumab in KRAS wild-type patients $(\mathrm{N}=58)$.

\begin{tabular}{cc}
\hline Imaging response per RECIST & Number (\%) \\
\hline CR & $3(5.2 \%)$ \\
PR & $7(12.1 \%)$ \\
SD & $26(44.8 \%)$ \\
PD & $13(22.4 \%)$ \\
Did not have imaging & $9(15.5 \%)$ \\
CEA response & $29(79.6 \%)$ \\
PFS (months) & $5.0(95 \%$ CI $3-6)$ \\
OS (months) & $9.0(95 \%$ CI $5-14)$ \\
\hline
\end{tabular}

${ }^{*} \mathrm{CEA}$ response as $\%$ of patients who marked for CEA. 


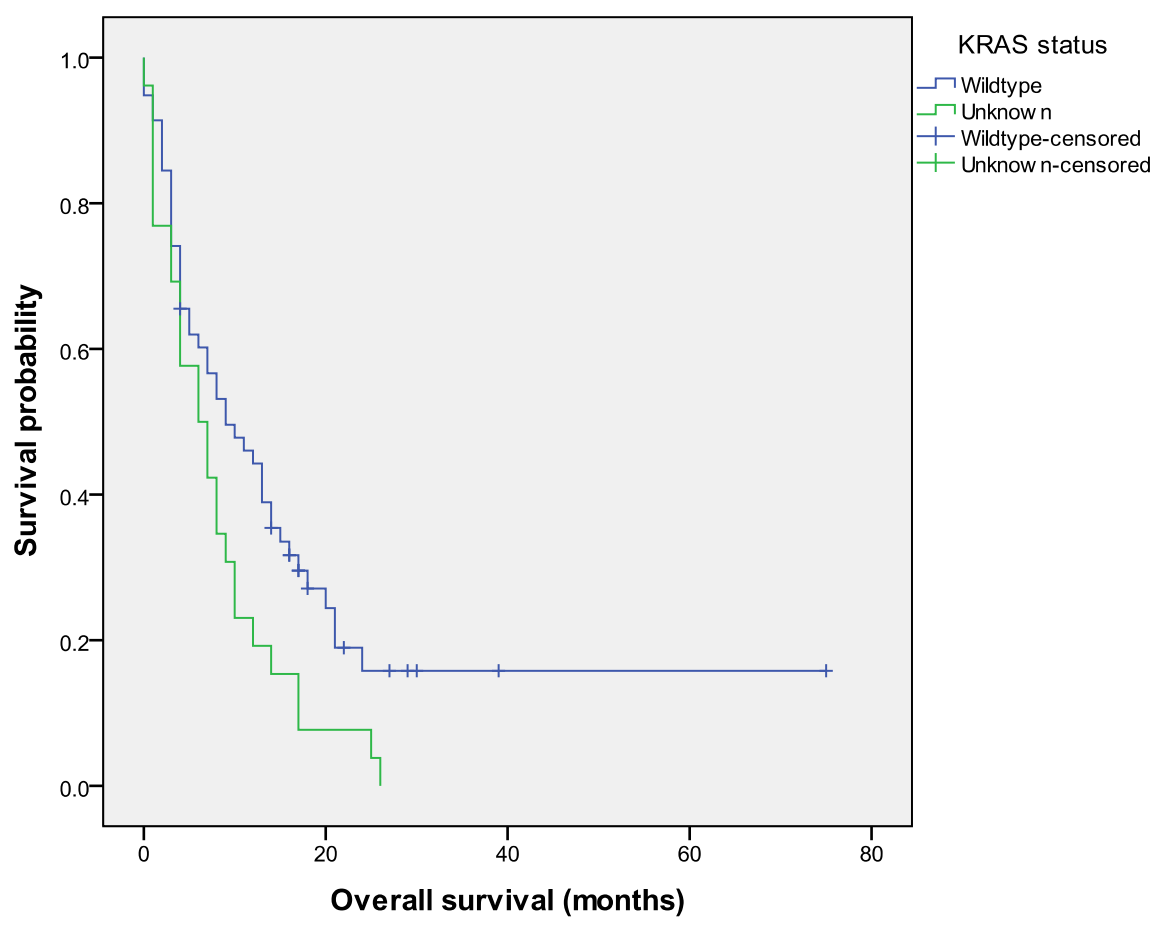

Figure 1. Overall survival in patients with KRAS wild-type tumors versus tumors with unknown KRAS status.

The two patients who were eventually found to have codon 12/13 KRAS mutations after being initiated on panitumumab did not have a response. The patient with a KRAS mutation in codon 8 remained on FOLFIRI/panitumumab for 4 cycles with stable disease followed by 4 cycles of 5-FU/panitumumab then 7 cycles of singleagent panitumumab before progressing. This patient remains alive today 50 months after initiating therapy.

\subsection{Predictors of Efficacy}

In a univariate analysis performed on patients with KRAS wild-type tumors only (see Table 3), the development of rash while on therapy strongly predicted an improved OS. Other predictors for OS in univariate analysis $(\mathrm{p}<0.1)$ included ECOG performance status $(0$ and 1 versus 2 or higher), panitumumab therapy with irinotecan or FOLFIRI versus single-agent panitumumab (see Figure 2) and peritoneal carcinomatosis versus other metastatic sites. The number of prior lines of therapy and age did not significantly impact PFS or OS. Cox-regression multivariate analysis with these covariates revealed that only ECOG performance status $(p=0.0013)$ had a statistically significant impact on OS.

Therapy choice with panitumumab similarly showed a trend towards better PFS with irinotecan (9.0 months, 95\% CI 3.0 - 14.0) when compared to with FOLFIRI (5.0 months, $95 \%$ CI $2.0-8.0$ ) or as single-agent (PFS 3.0 months $95 \%$ CI 0.0 - 5.0) although differences were not statistically significant $(\mathrm{p}=0.190)$ in univariate analysis.

\subsection{Prior Cetuximab Therapy}

Seventeen (19.5\%) patients had received cetuximab prior to starting panitumumab. For $8(47.1 \%)$ patients the tumors were known to be KRAS wild-type, for $9(52.9 \%)$ of them the KRAS mutational status was unknown. Five patients had cetuximab therapy discontinued due to toxicities. For the 12 patients who received cetuximab until progression, seven patients $(58.3 \%)$ had a clinical benefit rate with cetuximab and 1 patient had a partial response on imaging studies. Of those 7 patients, 5 (71\%) subsequently had clinical benefit ( 1 response, 6 with stable disease) from panitumumab therapy while only $1(25 \%)$ of 4 patients who did not have clinical benefit with cetuximab did so with panitumumab. Two of the 5 patients who benefitted received panitumumab as single agent while the other 3 had combination therapy.

\subsection{Panitumumab Administration}

Patients received a median number of 3 cycles (range 0.5 - 21 cycles). Eleven patients had two different regimens with panitumumab. Four patients had panitumumab started as a single agent and chemotherapy was added with irinotecan (2) or FOLFIRI (2) upon progression with stabilization of disease for 4 - 6 months in all four cases.

Four patients received panitumumab in combination with FOLFIRI and had partial or complete responses to therapy and were subsequently switched to single-agent panitumumab (2), 5-FU and panitumumab (1), or had therapy discontinued (1). The patient who had therapy 
Table 3. Univariate analysis of predictors of overall survival in KRAS wild-type patients $(\mathbf{N}=58)$.

\begin{tabular}{|c|c|c|c|c|}
\hline & $\mathrm{n}$ & Median OS (mo) & $95 \% \mathrm{CI}$ & $\mathrm{p}$-value \\
\hline \multicolumn{5}{|l|}{ Rash } \\
\hline Present & 30 & 14 & $8-21$ & \multirow{2}{*}{0.002} \\
\hline Not present & 28 & 4 & $3-10$ & \\
\hline \multicolumn{5}{|l|}{ Performance status } \\
\hline ECOG 0 & 8 & 17 & $3-21$ & \multirow{3}{*}{$<0.0001$} \\
\hline ECOG 1 & 37 & 13 & $8-18$ & \\
\hline ECOG 2 or higher & 13 & 2 & $0-3$ & \\
\hline \multicolumn{5}{|l|}{ Metastatic site } \\
\hline Liver or lung limited & 18 & 12.5 & $4-17$ & \multirow{3}{*}{0.085} \\
\hline$>1$ site & 32 & 11 & $4-18$ & \\
\hline Peritoneal carcinomatosis & 8 & 3 & $0-8$ & \\
\hline \multicolumn{5}{|l|}{ Panitumumab administration ${ }^{*}$} \\
\hline Single agent & 16 & 7 & $1-13$ & \multirow{3}{*}{0.04} \\
\hline With FOLFIRI & 27 & 9 & $4-17$ & \\
\hline With irinotecan & 11 & 24 & $4-\mathrm{N} / \mathrm{A}$ & \\
\hline \multicolumn{5}{|l|}{ Age } \\
\hline$\leq 61$ & 34 & 11 & $6-14$ & \multirow{2}{*}{0.283} \\
\hline$>61$ & 24 & 7 & $3-16$ & \\
\hline \multicolumn{5}{|c|}{ Number of previous chemotherapy regimens } \\
\hline None & 3 & Not reached & $8-\mathrm{N} / \mathrm{A}$ & \multirow{4}{*}{0.414} \\
\hline 1 & 23 & 7 & $1-13$ & \\
\hline 2 & 26 & 10 & $5-15$ & \\
\hline 3 or more & 6 & 4 & $0-18$ & \\
\hline
\end{tabular}

*3 patients got panitumumab with FOLFOX and 1 with capecitabine; they are not included in this analysis.

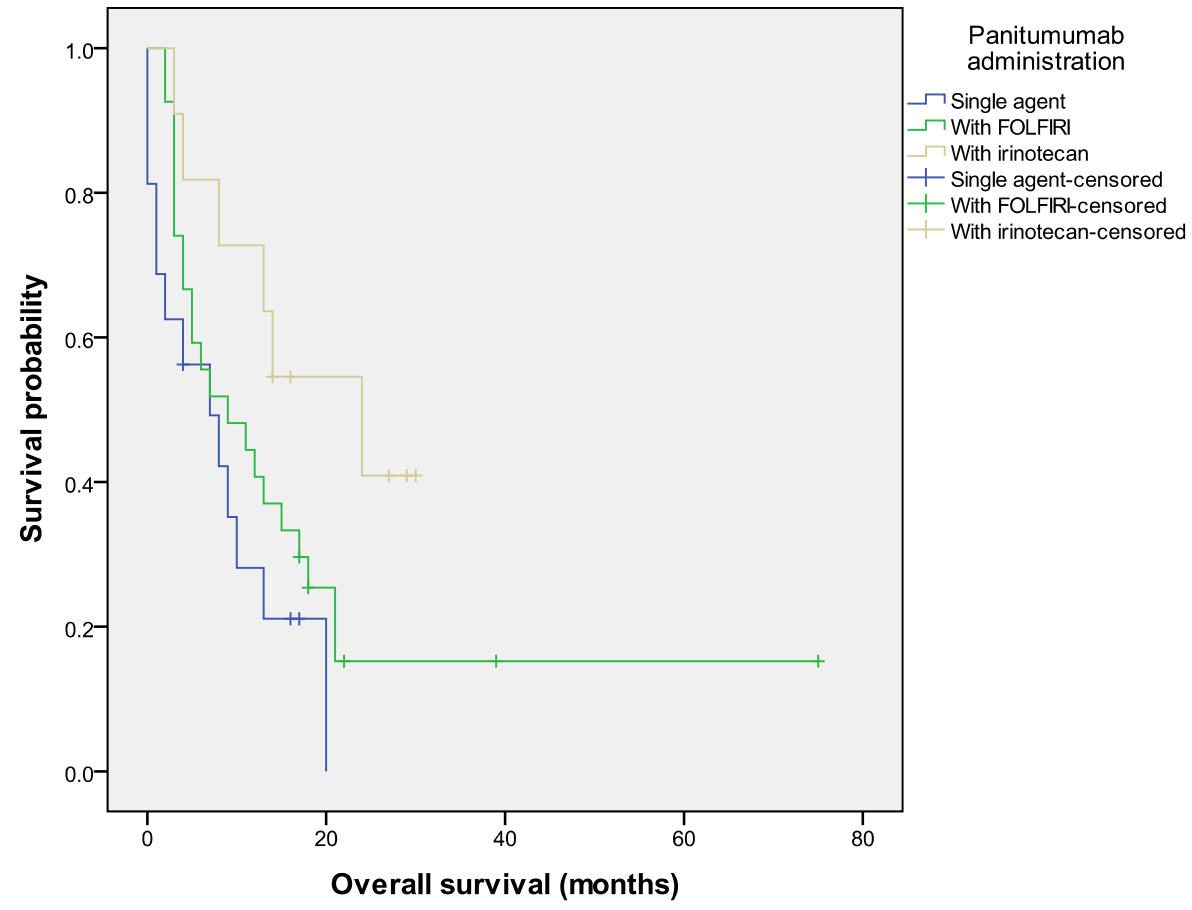

Figure 2. Overall survival in patients with KRAS wild-type tumors grouped by how panitumumab was administered. 
stopped had recurrence of disease on two separate occasions and FOLFIRI/panitumumab was restarted with a complete response on the 2 nd recurrence and a partial response on the $3 \mathrm{rd}$ recurrence. He remained on therapy for a total of 21 cycles with breaks after responses before eventually progressing.

Seventeen patients (19.5\%) received less than 2 cycles (4 doses) of panitumumab before having therapy stopped for progression of disease (16 patients), and one patient developed a pneumothorax.

Therapy was stopped because of toxicities in 4 (4.6\%) patients, due to progression in $76(87.4 \%)$ patients, due to no evidence of disease in $3(3.4 \%)$ patients and for other reasons in $4(4.6 \%)$ patients ( 1 to get treatment for a 2nd primary cancer, 1 for pneumothorax development and 1 for metastatectomy during which the patient died, 1 was undetermined).

\subsection{Toxicities}

Toxicities were consistent with previously published studies with skin rash (45\%), fatigue $(13 \%)$, mucositis $(8 \%)$ and diarrhea $(6 \%)$ being most commonly reported. All patients started at a dose of $6 \mathrm{mg} / \mathrm{kg}$ every other week but $13(14.9 \%)$ patients needed a dose reduction by $25 \%$ - 50\% for toxicities: 10 patients had grade 3 rash, 1 patient had mucositis and 1 patient had mucositis and diarrhea. One patient had an infusion reaction to cetuximab but tolerated panitumumab without complications.

\section{Discussion}

In this study we found a response rate of $17 \%$ to panitumumab therapy which is similar to prior phase III study results when panitumumab is given in the refractory setting [4]. All of these patients were known to have KRAS wild-type tumors which is predictive of response to EGFR inhibitors. A total of 36 out of 58 (62.1\%) patients with KRAS wild-type tumors had a clinical benefit from therapy and showed an improved OS compared to patients with unknown KRAS tumor status and this led us to restrict our univariate analysis to patients with known KRAS wild-type tumors. In this study, panitumumab was most commonly given with FOLFIRI and there was some evidence that therapy can be de-escalated to a single agent in patients with objective responses and escalated again with good efficacy upon progression.

The number of prior therapy lines did not impact PFS or OS which suggests that EGFR inhibitors retain their efficacy even in the 2 nd or 3 rd line setting. Although the number of patients in our analysis is small, these findings suggest it may be wise to reserve EGFR inhibitors until after progression on regimens containing anti-angiogenic agents.

Three variables had a significant impact on OS in uni- variate analysis among patients with KRAS wild-type tumors; performance status, rash and type of chemotherapy combination with panitumumab. About $24 \%$ of patients had a performance status of $2-3$ and more than half of them received panitumumab as a single agent. They did have a significantly shorter PFS and OS which is not surprising and has been demonstrated in other colorectal cancer trials [15]. It is important to note that panitumumab as a single agent, which is considered to be better tolerated than when the agent is administered with cytotoxic therapy, did not seem to benefit patients who have a poor performance status and should not be implemented.

The development of a rash was significantly associated with a better OS which is in concordance with other studies which have associated grade 2 - 4 skin toxicities with a significantly longer OS (hazard ratio $0.60, \mathrm{p}=$ 0.0033 ) compared to patients with grade 1 skin toxicities [16].

Patients receiving panitumumab with irinotecan had better PFS and OS than patients receiving panitumumab as a single-agent and this regimen was equivalent to or better than pairing FOLFIRI with panitumumab. All but 2 of these patients had been on irinotecan-based therapy prior to starting panitumumab. In the BOND trial, patients who had previously progressed on irinotecan-based chemotherapy had an ORR of $22 \%$, a PFS of 4.1 months and OS of 8.6 months when treated with irinotecan and cetuximab [17]. Preclinical evidence suggests that an EGFR inhibitor may re-sensitize cell lines to irinotecan after they become irinotecan-resistant [18]. This data further supports reserving EGFR inhibitors until the 2nd or 3rd line.

Sites of metastasis did not reach statistical significance but it is notable that patients with peritoneal carcinomatosis did very poorly with a median OS of 3 months. Peritoneal carcinomatosis was recently shown to be associated with poor survival in a pooled analysis of the N9741 and N9841 trials [19].

Interestingly, one of our patients had an atypical KRAS mutation in codon 8 (V8I) within exon 2 and was started on panitumumab as 3 rd line therapy. The patient did have prolonged stabilization of disease for 17 months and is still alive 50 months after initiating therapy. Not all KRAS mutations seem to carry the same predictive value for EGFR inhibitor efficacy as recently demonstrated with the G13D mutation in codon 13 within exon 2 [20] and our patient certainly appeared to benefit from panitumumab.

The analysis of patients on cetuximab prior to panitumumab is limited by the fact that over half had tumors with unknown KRAS mutational status. It did show that patients who did not have clinical benefit from cetuximab in the past were unlikely to benefit from panitumu- 
mab. However, $71 \%$ of patients who did have clinical benefit from cetuximab did so with panitumumab. A recently published study found $20 \%$ (2 of 10 ) of patients who had progressed on cetuximab to have tumors with an acquired EGFR ectoderm mutation, rendering cetuximab unable to bind to the receptor while the acquired mutation did not affect the binding of panitumumab [21]. It is therefore possible that patients who have previously progressed on cetuximab could benefit from panitumumab and our study suggests that this would be limited to patients who have previously benefitted from cetuximab.

There are a number of limitations to our study. KRAS mutational status was not assessed in any patients starting panitumumab prior to June 2008 as it did not become an NCCN recommendation until November 2008 and was therefore unknown in 26 (29.9\%) patients. We therefore did not include these patients in our univariate and multivariate analysis of predictors of efficacy. The retrospective design allows for a number of biases including the potential for selection and recall bias, although we did include all patients who received at least 1 dose of panitumumab. There are limitations to accurately collecting data on toxicities and numeric grading was usually not available on chart review. Furthermore, imaging studies might not have been done at regular 2 - 3-month intervals in all patients allowing for a potential overestimation of PFS in some cases.

In summary, our study suggests that panitumumab might best be reserved for use as 2nd or 3rd line therapy rather than moving it up to 1 st line as the number of prior therapies does not seem to affect its efficacy. It also suggests that EGFR inhibitor therapy does not benefit patients who have poor performance status ( 2 or above) any more than cytotoxic chemotherapies do. It furthermore reminds us that not all KRAS mutations are the same, suggesting that codon 8 mutations may not affect efficacy of EGFR inhibitors. Finally, patients who have previously progressed on cetuximab could possibly benefit from panitumumab if they had clinical benefit from cetuximab therapy.

\section{REFERENCES}

[1] R. Siegel, E. Ward, O. Brawley and A. Jemal, "Cancer Statistics, 2011: The Impact of Eliminating Socioeconomic and Racial Disparities on Premature Cancer Deaths," CA: A Cancer Journal for Clinicians, Vol. 61, No. 4, 2011, pp. 212-236. doi:10.3322/caac.20121

[2] H. K. Sanoff, D. J. Sargent, M. E. Campbell, R. F. Morton, C. S. Fuchs, R. K. Ramanathan, et al., "Five-Year Data and Prognostic Factor Analysis of Oxaliplatin and Irinotecan Combinations for Advanced Colorectal Cancer: N9741," Journal of Clinical Oncology, Vol. 26, No. 35, 2008, pp. 5721-5727. doi:10.1200/JCO.2008.17.7147

[3] A. Lievre, J. B. Bachet, D. Le Corre, V. Boige, B. Landi,
J. F. Emile, et al., "KRAS Mutation Status Is Predictive of Response to Cetuximab Therapy in Colorectal Cancer," Cancer Research, Vol. 66, No. 8, 2006, pp. 3992 3995. doi:10.1158/0008-5472.CAN-06-0191

[4] R. G. Amado, M. Wolf, M. Peeters, E. Van Cutsem, S. Siena, D. J. Freeman, et al., "Wild-Type KRAS Is Required for Panitumumab Efficacy in Patients with Metastatic Colorectal Cancer," Journal of Clinical Oncology, Vol. 26, No. 10, 2008, pp. 1626-1634.

doi:10.1200/JCO.2007.14.7116

[5] M. Peeters, T. J. Price, A. Cervantes, A. F. Sobrero, M. Ducreux, Y. Hotko, et al., "Randomized Phase III Study of Panitumumab with Fluorouracil, Leucovorin, and Irinotecan (FOLFIRI) Compared with FOLFIRI Alone as Second-Line Treatment in Patients with Metastatic Colorectal Cancer," Journal of Clinical Oncology, Vol. 28, No. 31, 2010, pp. 4706-4713. doi:10.1200/JCO.2009.27.6055

[6] J. Y. Douillard, S. Siena, J. Cassidy, J. Tabernero, R. Burkes, M. Barugel, et al., "Randomized, Phase III Trial of Panitumumab with Infusional Fluorouracil, Leucovorin, and Oxaliplatin (FOLFOX4) versus FOLFOX4 Alone as First-Line Treatment in Patients with Previously Untreated Metastatic Colorectal Cancer: The PRIME Study," Journal of clinical oncology, Vol. 28, No. 31, 2010, pp. 4697-4705. doi:10.1200/JCO.2009.27.4860

[7] T. S. Maughan, R. A. Adams, C. G. Smith, A. M. Meade, M. T. Seymour, R. H. Wilson, et al., "Addition of Cetuximab to Oxaliplatin-Based First-Line Combination Chemotherapy for Treatment of Advanced Colorectal Cancer: Results of The Randomised Phase 3 MRC COIN Trial," Lancet, Vol. 377, No. 9783, 2011, pp. 2103-2114. doi:10.1016/S0140-6736(11)60613-2

[8] K. M. Tveit, T. Guren, B. Glimelius, P. Pfeiffer, H. Sorbye, S. Pyrhonen, et al., "Phase III Trial of Cetuximab with Continuous or Intermittent Fluorouracil, Leucovorin, and Oxaliplatin (Nordic FLOX) versus FLOX Alone in First-Line Treatment of Metastatic Colorectal Cancer: The NORDIC-VII Study," Journal of Clinical Oncology, Vol. 30, No. 15, 2012, pp. 1755-1762. doi:10.1200/JCO.2011.38.0915

[9] J. Taieb, T. S. Maughan, C. Bokemeyer, E. Van Cutsem, T. Brodowicz, G. Folprecht, et al., "Cetuximab Combined with Infusional 5-Fluorouracil/Folinic Acid (5-FU/FA) and Oxaliplatin in Metastatic Colorectal Cancer (mCRC): A Pooled Analysis of COIN and OPUS Study Data," Journal of Clinical Oncology, Vol. 30, 2012, Abstract 3574 .

[10] J. Metges, J. Raoul, N. Achour, O. Capitain, A. Gourlaouen, J. Ramee, et al., "PANERB Study: Panitumumab after Cetuximab-Based Regimen Failure," Journal of Clinical Oncology, Vol. 28, No. 15, 2010, p. e14000.

[11] R. C. Wadlow, A. F. Hezel, T. A. Abrams, L. S. Blaszkowsky, C. S. Fuchs, M. H. Kulke, et al., "Panitumumab in Patients with KRAS Wild-Type Colorectal Cancer after Progression on Cetuximab," The Oncologist, Vol. 17, No. 1, 2012, pp. e27-e34.

[12] M. W. Saif, K. Kaley, E. Chu and M. S. Copur, "Safety and Efficacy of Panitumumab Therapy after Progression with Cetuximab: Experience at Two Institutions," Clini- 
cal Colorectal Cancer, Vol. 9, No. 5, 2010, pp. 315-318. doi:10.3816/CCC.2010.n.046

[13] D. Power, M. Shah, T. Asmis, J. Garcia and N. Kemeny, "Safety and Efficacy of Panitumumab Following Cetuximab: Retrospective Review of the Memorial Sloan- Kettering Experience," Investigational New Drugs, Vol. 28, No. 3, 2010, pp. 353-360. doi:10.1007/s10637-009-9268-y

[14] E. A. Eisenhauer, P. Therasse, J. Bogaerts, L. H. Schwartz, D. Sargent, R. Ford, et al., "New Response Evaluation Criteria in Solid Tumours: Revised RECIST Guideline (Version 1.1)," European Journal of Cancer, Vol. 45, No. 2, 2009, pp. 228-247. doi:10.1016/j.ejca.2008.10.026

[15] D. J. Sargent, C. H. Köhne, H. K. Sanoff, B. M. Bot, M. T. Seymour, A. de Gramont, et al., "Pooled Safety and Efficacy Analysis Examining the Effect of Performance Status on Outcomes in Nine First-Line Treatment Trials Using Individual Data from Patients with Metastatic Colorectal Cancer," Journal of Clinical Oncology, Vol. 27, No. 12, 2009, pp. 1948-1955. doi:10.1200/JCO.2008.20.2879

[16] M. Peeters, S. Siena, E. Van Cutsem, A. Sobrero, A. Hendlisz, S. Cascinu, et al., "Association of ProgressionFree Survival, Overall Survival, and Patient-Reported Outcomes by Skin Toxicity and KRAS Status in Patients Receiving Panitumumab Monotherapy," Cancer, Vol. 115, No. 7, 2009, pp. 1544-1554. doi:10.1002/cncr.24088

[17] D. Cunningham, Y. Humblet, S. Siena, D. Khayat, H. Bleiberg, A. Santoro, et al., "Cetuximab Monotherapy and Cetuximab plus Irinotecan in Irinotecan-Refractory Metastatic Colorectal Cancer," New England Journal of Medicine, Vol. 351, No. 4, 2004, pp. 337-345. doi:10.1056/NEJMoa033025

[18] M. Yashiro, H. Qiu, T. Hasegawa, X. Zhang, T. Matsuzaki and K. Hirakawa, "An EGFR Inhibitor Enhances the Efficacy of SN38, an Active Metabolite of Irinotecan, in SN38-Refractory Gastric Carcinoma Cells," British Journal of Cancer, Vol. 105, No. 10, 2011, pp. 1522-1532. doi:10.1038/bjc. 2011.397

[19] J. Franko, Q. Shi, C. D. Goldman, B. A. Pockaj, G. D. Nelson, R. M. Goldberg, et al., "Treatment of Colorectal Peritoneal Carcinomatosis with Systemic Chemotherapy: A Pooled Analysis of North Central Cancer Treatment Group Phase III Trials N9741 and N9841," Journal of Clinical Oncology, Vol. 30, No. 3, 2011, pp. 263-267.

[20] S. Tejpar, I. Celik, M. Schlichting, U. Sartorius, C. Bokemeyer and E. Van Cutsem, "Association of KRAS G13D Tumor Mutations with Outcome in Patients with Metastatic Colorectal Cancer Treated with First-Line Chemotherapy with or Without Cetuximab," Journal of Clinical Oncology, Jun 252012 [Epub Ahead of Print].

[21] C. Montagut, A. Dalmases, B. Bellosillo, M. Crespo, S. Pairet, M. Iglesias, et al., "Identification of a Mutation in the Extracellular Domain of the Epidermal Growth Factor Receptor conferring Cetuximab Resistance in Colorectal Cancer," Nature Medicine, Vol. 18, No. 9, 2012, pp. 14451445. doi:10.1038/nm0912-1445b 